\title{
Towards Low-Carbon Conferencing: Acceptance of Virtual Conferencing Solutions and Other Sustainability Measures in the ALIFE Community
}

\author{
Harold Fellermann ${ }^{1}$, Alexandra S. Penn ${ }^{2}$, Rudolf M. Füchslin ${ }^{3}$, Jaume Bacardit ${ }^{1}$ and Angel Goñi-Moreno ${ }^{1}$ \\ ${ }^{1}$ School of Computing, Newcastle University, Newcastle Upon Tyne, UK \\ ${ }^{2}$ Centre for Environmental Strategy, University of Surrey, Guildford, UK \\ ${ }^{3}$ School of Engineering, Zurich University of Applied Sciences, Winterthur, Switzerland \\ harold.fellermann@newcastle.ac.uk, angel.goni-moreno@newcastle.ac.uk
}

\begin{abstract}
The latest report from the Intergovernmental Panel on Climate Change (IPCC) estimated that humanity has a time window of about 12 years in order to prevent anthropogenic climate change of catastrophic magnitude. Green house gas emission from air travel, which is currently rising, is possibly one of the factors that can be most readily reduced. Within this context, we advocate for the re-design of academic conferences in order to decrease their environmental footprint. Today, virtual technologies hold the promise to substitute many forms of physical interactions and increasingly make their way into conferences to reduce the number of travelling delegates. Here, we present the results of a survey in which we gathered the opinion on this topic of academics worldwide. Results suggest there is ample room for challenging the (dangerous) business-as-usual inertia of scientific lifestyle.
\end{abstract}

\section{Background}

The latest report from the Intergovernmental Panel on Climate Change (IPCC) estimated that humanity has a time window of about 12 years in order to prevent anthropogenic climate change of catastrophic magnitude (IPCC, 2018) and calls for drastic actions in order to halve $\mathrm{CO}_{2}$ emissions by no later than 2030. In light of these reports, a growing number of scientists rethinks their way of working and attempts to implement a low-carbon agenda (Nevins, 2014; Nathans and Sterling, 2016; Cobb et al., 2018).

As analyzed by Achten et al. (2013), professional mobility constitutes about $75 \%$ of the carbon emissions of a representative $\mathrm{PhD}$ project, with $35 \%$ of total emissions being associated with conference attendence alone.

Global impact of flying is immense-and it is rapidly increasing. Recent reports establish aviation impact as high as $2.1 \%$ of global $\mathrm{CO}_{2}$ emissions (Girling et al., 2018)roughly equivalent to Germany's total emissions. Moreover, this figure does not consider the enhanced impact of greenhouse gas emissions at high altitude (Lee et al., 2009).

Universities are also dealing with the problem of aviation (Nature editorial, 2015). For example, the University of British Columbia has recently published a comprehensive analysis of emissions due to flying - where conferences are highlighted as the biggest contributor to the academic environmental footprint (Wynes and Donner, 2018). University of California Los Angeles applies a tax to academic flights-although this measure "doesn't go too far" (Hasan, 2018). In addition to this, the University of Basel is considering mandatory train travel within some radius (LeyboldJohnson, 2019). However, university-sustainability policies do not necessarily tackle this issue and may "unintentionally encourage academic staff to fly more rather than less" (Glover et al., 2017).

Already almost a decade ago, a multi-hub conference (i.e., talks/audience distributed among different locations) paved the way for low-carbon conferencing strategies (Krumdieck and Orchard, 2011).

Here, we present the result of a community survey on the acceptance of different measures for implementing lowcarbon conferencing with particular emphasis on virtual conferencing solutions. The survey has been performed for the Artificial Life (ALIFE) community. Results may be considered in the future to adapt the ALIFE Conference to a low-carbon path.

\section{Results}

Our online survey consisted of 28 questions that assessed respondents general conferencing habits, their experience with and evaluation of virtual technologies to substitute for presential conferencing, as well as acceptance of potential measures that conference organizers might put in place to reduce the ecological impact of their event. The survey was open to unrestricted anonymous participation over a period of four weeks and had been announced through the mailinglist and Twitter feeds of the International Society of Artificial Life (announce@isal.groups.io), as well as the personal professional networks (email and Twitter) of the ALIFE 2019 organizers.

\section{Survey participants}

At the time of writing, the survey was completed by 170 respondents. Figure 1 shows the profile of respondents. Most of these work in academia (academic or student), 67\% are 

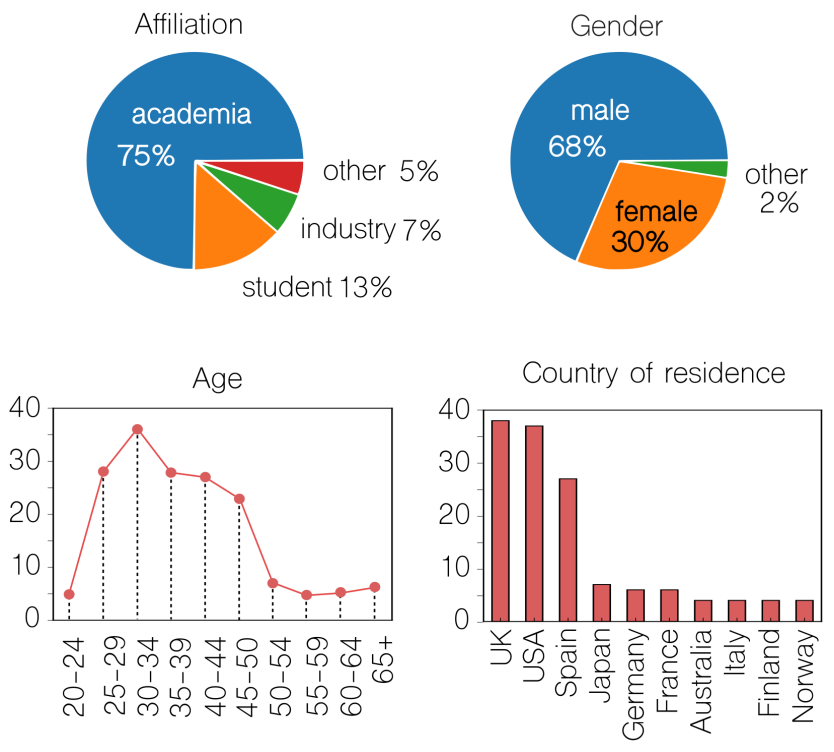

Figure 1: Demographics of survey respondents: professional affiliation, gender, age, and top-most countries of residence $(80 \%$ of total).

male and the majority is within 30 and 50 years of age.

Since the survey was openly accessible, we devised several question in order to determine respondents' affiliation with the Artificial Life community. Participants where asked whether they are interested in Artificial Life (very much: 68, somewhat: 48, not at all: 49), are working in Artificial Life (fully: 26, partly: 63, not at all: 77), have attended ALIFE or ECAL conferences (once: 10, 2-5 times: 32, 6-9 times: 14, more than 10 times: 5 , never: 105 ), have publised in the Artificial Life journal (once: 12, 2-5 times: 32, 6-9 times: 10, more than 10 times: 6, never: 107), or whether they are members of the International Society for Artificial Life (yes: 27, no: 130, do not know: 11). Since all of these are exceptedly correlated, we simply chose the answer to the question "Do you consider yourself as working in the field of Artificial Life" as an indicator of the community affiliation. With this, our survey includes 89 respondents (52\%) from the ALIFE community and 81 respondents (45\%) from other research areas, which includes 5 respondents who did not answer the respective question.

\section{Conference attendance}

Participants reported to attend an average of three to four conferences per year, one or two of which typically being intercontinental. All in all, conference attendence appears to follow a Poisson distribution, i.e. we do not observe a heavy tail distribution in our data sample (Figure 2).

Participants were asked to weigh their reasons for attending conferences (Figure 3). This establishes the priorities of the respondents, which eventually need to be considered

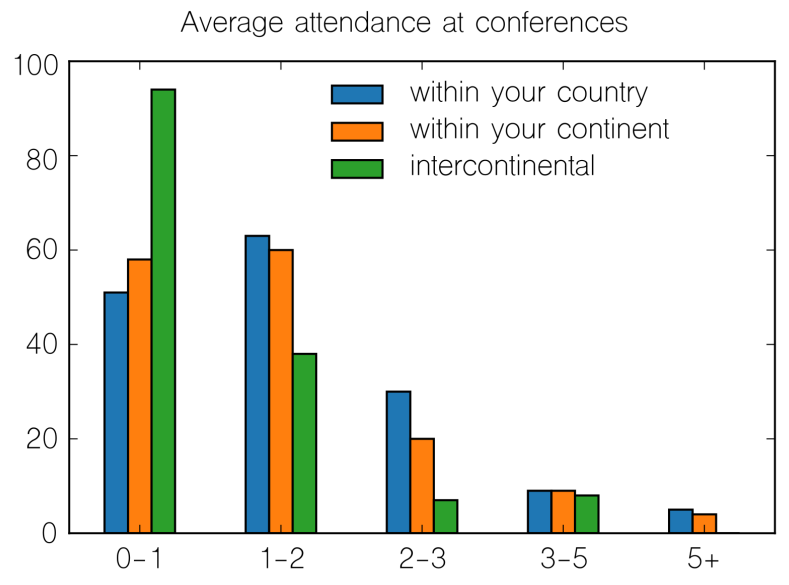

Figure 2: Average number of conferences attended per year.

when devising sustainability measures, particularly regarding inclusion of virtual remote participation. Presenting own work, discovering new work, and catching up with work done by colleagues were named as the three top priorities. Employment considerations such as finding potential future employers or employees where regarded less relevant. Additional comments allowed respondents to name further reasons, of which the following received the most mentions: Keeping in touch with friends and colleagues; boosting academic reputation, e.g. through invited talks; being provided with an environment that allows to completely focus on science and develop new ideas; travelling to new places, especially when paired with vacations.

Participants were then asked to think about the last conference they intended to attend, but eventually decided against. The main reasons for not attending were, in decreasing order: too much travel involved (85 mentions), travel budget exceeded (85 mentions), nothing to present (55), and competing professional (53 mentions) or personal commitments (42 mentions). Noteworthily, additional comments completed this list with five mentions of climate impact as a motif for not attending the conference in question.

If the conference in question would have allowed for virtual remote participation, survey respondents reported that they would have made use of this opportunity in order to: follow broadcast contributions (136 mentions), present own work as talk or poster (99 mentions), and discuss presentations in comments or forums (85 mentons). Comparably fewer people stated that they would have engaged in plenary or one-to-one video conversations (53 and 47 mentions, respectively). Only 29 respondents would have advertised job openings in forums or chat rooms. However, this number might reflect the relative scarceness of job opportunities more than the respondents willingness to advertise those. 
Main reasons for conference attendence

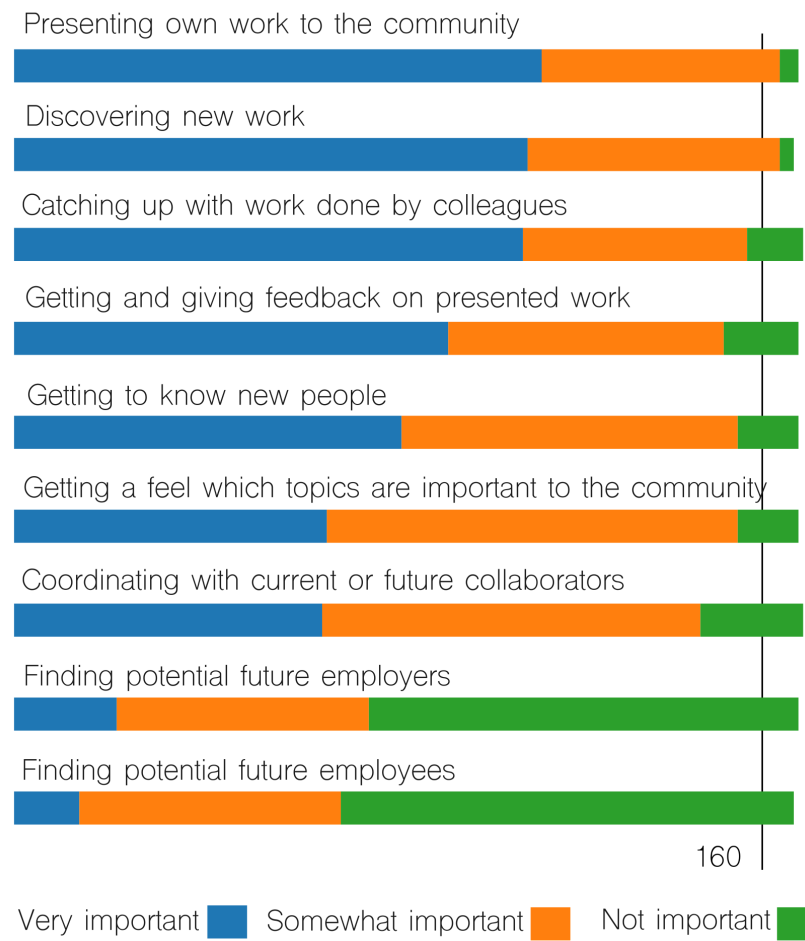

Figure 3: Main reasons to attend scientific conferences.

\section{Evaluation of virtual conferencing solutions}

The majority of survey respondents $(66 \%)$ have never attended a conference that was delivered virtually or had a significant portion of remotely delivered content. Yet, $62 \%$ reported that they did participate in webinar series and the vast majority $(92 \%)$ reports that they occasionally or regularly follow video lectures and/or recorded talks related to their field of work.

The majority of respondents $(69 \%)$ indicated that they would only be interested in attending a conference virtually at significantly reduced fee, while $14 \%$ were willing to pay a slightly reduced fee, and another $16 \%$ would only participate virtually if it were for free.

Participants were asked to evaluate (from strong advantage to strong disadvantage) the effects of virtual remote participation on different aspects of conferencing (Figure 4). The top advantages are money and time saving, the ability to reach a wider audience and to accommodate for conflicting professional or personal duties. All these advantages are deemed about equally strong. Additional comments added the following benefits: Reduced climate impact (31 mentions); no travel inconvenience or visa problems (5 mentions); longer availability of recorded talks (5 mentions); improved accessibility for delegates with disabilities (4 mentions).

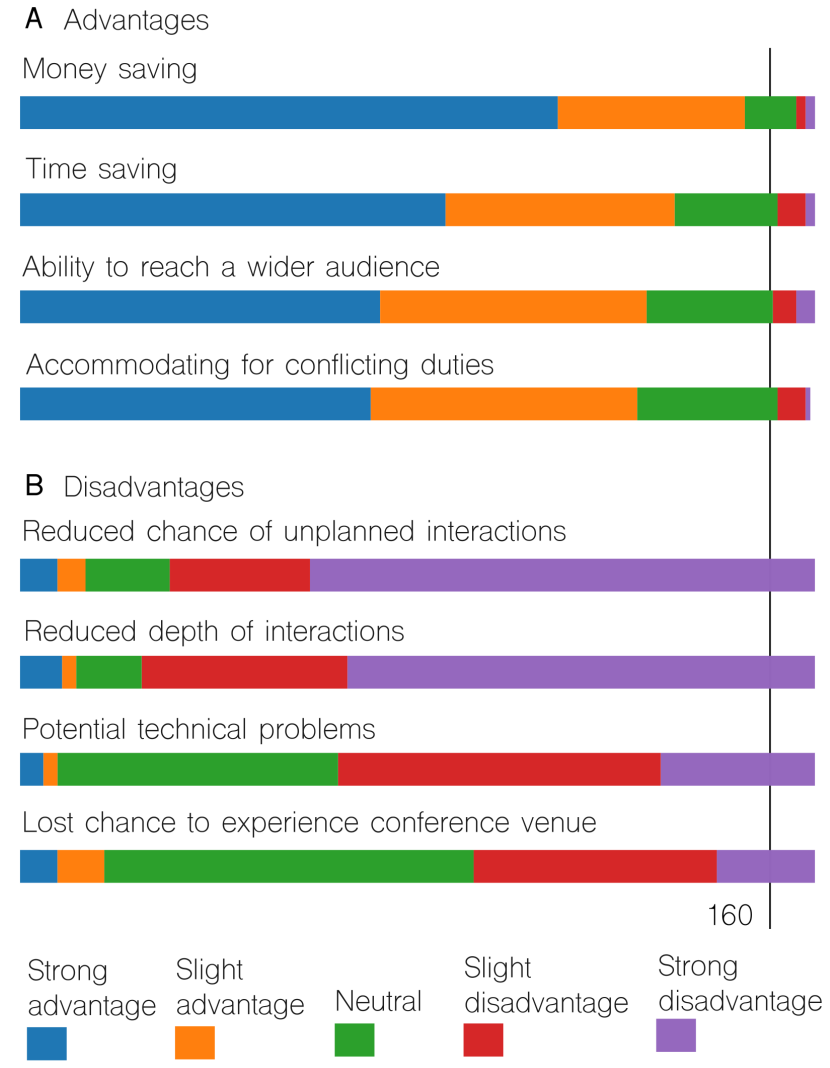

Figure 4: Advantages and disadvantages of virtual conferencing.

The top disadvantages are the lost chance of unplanned interactions, and reduced depth of interaction. Potential technical problems are deemed as less significant, as is the lost chance to experience the conference location. Additional comments added the following concerns: virtual respondents miss out on networking opportunities, including after hour activities (11 mentions); harder for virtual participants to fully commit to the event (10 mentions); remote presentations being less engaging for presential delegates ( 3 mentions); Another important concern was that broadcasting and recording of talks discourages presentation of work-inprogress and unpublished material, as well as open discussion about presented work.

The survey then asked respondents to evaluate how well virtual conference solutions can deliver the aspects of scientific conferences (from "much better" to "much worse"). Results are shown in Figure 5. As expected, virtual solution are deemed to perform well (in some cases even better than presential conferencing) when it comes to delivering technical content (seeing what is important to the community, discovering new work, presenting own work), but fall short when it comes to supporting social interaction (finding potential future employers or employees, coordinating with 
Suitability of virtual conferencing solutions

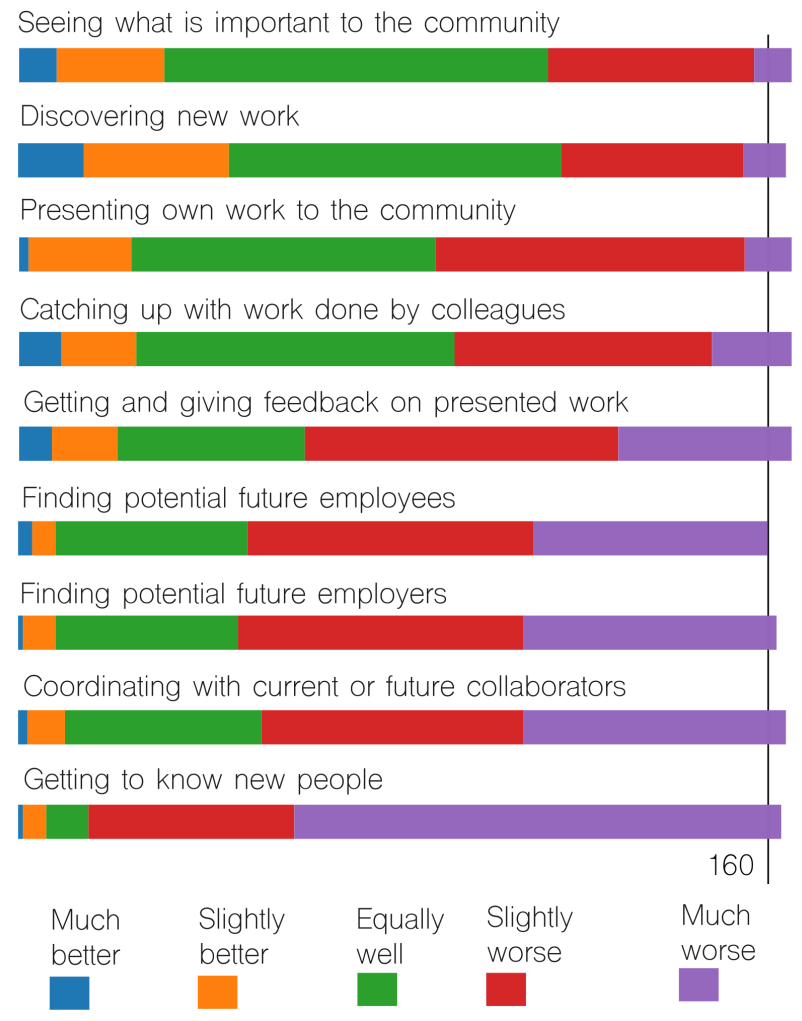

Figure 5: How well can virtual technologies deliver different aspects of conferences compared to presential participation?

collaborators, and getting to know new people).

Interestingly, when crossing these answers with the ones to the question on the importance of various conferencing aspects, it can be concluded that the functions that are seen as most important (see Figure 3) are also deemed to be delivered equally well or only slightly worse by virtual conferencing technologies compared to presential conference attendance.

The survey also included several open questions about respondents' experience with virtual conferencing technologies and asked what they saw that worked particularly well, what could have been improved where things went wrong, and where they see the biggest barriers that would need to be overcome in order to make virtual conferencing an equal or superior option. Feedback from these questions generally fell into three categories: technical, organizational, and social.

On the technical side, the software platform used for broadcasting and/or video conferencing is crucial as it has been responsible for many of the reported problems. If remote participants are to interact virtually with the presential audience, virtual conferencing solutions should not require installation of proprietary clients, particularly if they are not equally well supported on all major operating systems. Internet connection, both video and audio equipment, but especially the latter, need to be of sufficient quality to not impede the conversation. Similarly, the choice of lightning can significantly influence virtual conferencing experience. Solutions need to be supervised by well trained technical support, and organizers should provide alternative ways of access and/or backup recordings if part of the technology becomes unreliable. If all requirements are met, several survey respondents feel that virtually delivered or received presentations can actually be superior to presential ones, as the former is also often affected by poor sound quality and visuals in the venue.

Regarding modes of presentation, respondents showed some divide as to whether life presentation or pre-recorded presentation leads to a better delivery of a remote talk. Life presentation is generally reported to be more engaging, but more susceptible to technical issues. In both cases, delivery of a talk to the camera is generally unsympathetic to people's audience-oriented delivery style, and the lack of audience feedback renders remote delivery less engaging. In a life setup, this might be addressed by streaming a feed of the audience to the presenter. However, lack of engagement is ultimately not a technical problem but is often due to the speaker himself not feeling present at the event. Several respondents advocate for pre-recorded presentations followed by life Q\&A sessions.

For virtual life interaction, special focus needs to be placed on enabling interaction between presential and virtual participants, as interactions can easily appear unsatisfying due to time lags and reduced communication bandwidth of virtual participation solutions. While some survey respondents advocate for questions and answers to be moderated by the chair (for example using technologies such as sli.do), other respondents prefer the opportunity for a direct dialogue with the presenter. A particular difficulty is to include virtual participants in workshops, where the focus lies on discussion rather than delivery of presentations. On the other hand, change between different interaction formats can also support the exchange of ideas and current affairs: Discussions that start in a Q\&A session or virtual chat can readily be followed up in specialized discussion forums, and virtual break-out sessions can reconvene in plenary.

Independent of virtual participation, most respondents valued the recording and streaming of presentations, both for their potential to reach a wider audience as well as for their longevity. The latter being especially valuable as conferences with parallel tracks do not allow for participants to follow the whole programme. On the flip-side, wide and long-lasting availability of conference contributions can make participants less willing to present work in progress and unpublished results, which can have a detrimental impact on the quality of the discussion.

In line with the numeric evaluation shown in Figure 5, 
the most mentioned biggest barrier that would need to be overecome in order to make virtual conferencing an equal or superior option to presential conferencing is the lack of serendipitous interactions, unplanned face-to-face conversations, and informal networking activities. This was most often expressed as the lack of "virtual hallway tracks", "virtual lunches" and after hour activities. No respondent hinted towards any existing technology with a potential to enable such interactions to a meaningful extent. As a consequence of falling short in this important conferencing function, several respondents expressed their worry that opening traditional conferences for virtual participation might introduce a two-class scientific society, where virtual participants (those who are unable or unwilling to fly more, presumably mainly early career scientists) are disadvantaged compared to presential attendants.

A recurrent suggestion that might compensate for the lack of social interactions in virtual conferences was to host scientific events in several parallel local venues, which are then linked up using virtual conferencing technology. This form of conferencing is able to create an opportunity for full immersion, social networking and direct physical interaction, while still reducing the amount of long distance air travel.

\section{Acceptance of sustainability measures}

Finally, we asked participants to evaluate different measures that conference organizers might install in order to reduce the ecological impact of their event (Figure 6). Most respondents strongly or slightly welcomed the inclusion of virtual remote talks and poster presentations, despite all difficulties expressed in the previous sections. In general, respondents demonstrate a higher acceptance of optional measures and incentives (such as registration discounts for train travel), whereas mandatory measures (such as exclusively vegetarian and sustainably sourced catering) received lower sanctioning, particularly when connected with increased registration fees. Carbon offsetting was ranked equally low (with the optional measure being slightly more accepted than the mandatory one), likely due to its controversial benefit. Installing presential participation quota meets the lowest acceptance by survey respondents, particularly when correlated with travel distance or when quota are installed per research group. In general, all but these last two sustainability measures received more supportive than opposing evaluations.

Participants were more inclined to accept hard presential participation quota when virtual participation is notably discounted (Figure 7). 61\% of the respondents would welcome enforced presential participation limits if connected with a significant registration discount and $72 \%$ would welcome the measure if virtual presentation were at no cost.

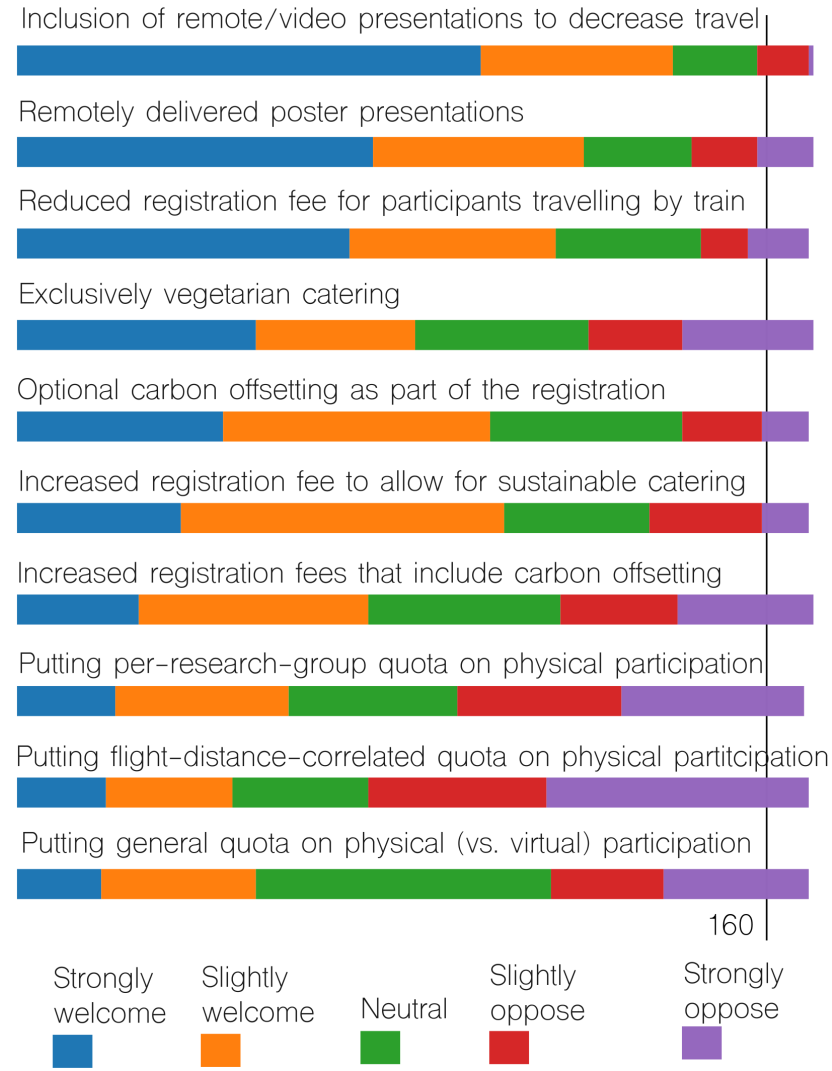

Figure 6: Acceptance of different sustainability measures for scientific conferences

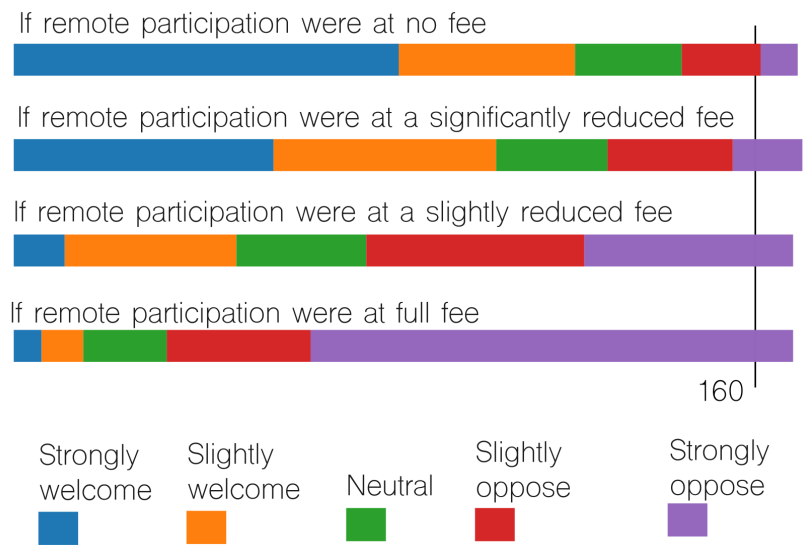

Figure 7: Acceptance of hard physical attendance quota for different registration schemes 


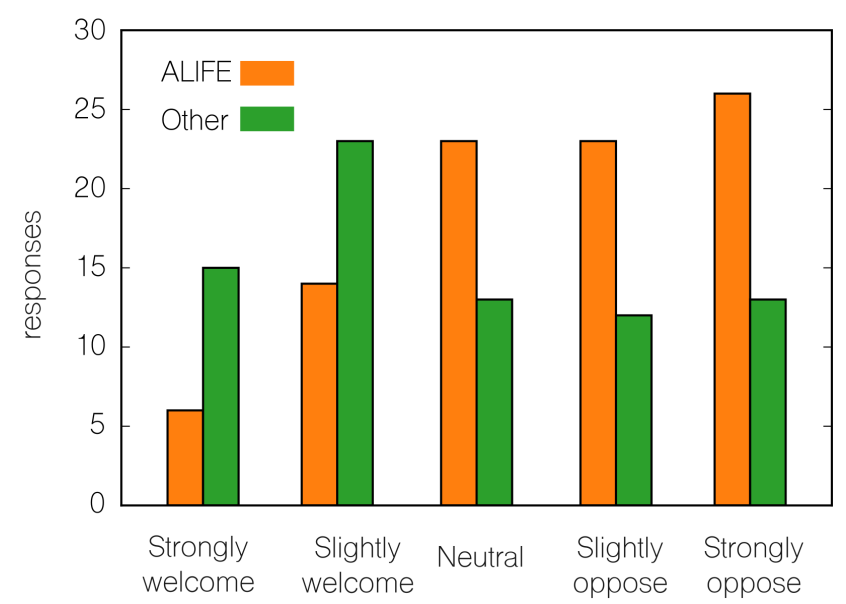

Figure 8: Different acceptance of flight-distance-correlated presential participation quota within the ALIFE community and other respondents.

\section{Difference between the ALIFE community and other survey respondents}

We have analyzed all survey results with respect to differences between responses from self-declared ALIFE community members against the rest of the respondents. For almost none of the questions could a significant difference be noticed.

The only exception is that ALIFE community members show a stronger opposition than other respondents towards conference organizers imposing flight-distance-correlated presential participation quota (Figure 8). This might be due to a number of reasons: Firstly, ALIFE is a small community that holds essentially one international conference per year. If people from certain origins are discouraged to participate presentially, they cannot be offered an alternative closer to their country of residence. Secondly, because the community is relatively small, there might be a stronger desire to physically co-locate all its members - something that is unfeasible for larger scientific communities. Thirdly, ALIFE is also a very diverse community. Co-locating its members might help to keep the community coherent and prevent the loss of exchange between subfields of Artificial Life. Lastly, we cannot exclude a sampling bias due to the way survey respondents had been recruited. If this were the case, it is nevertheless interesting that such bias shows most strongly when it comes to this particular sustainability measure.

\section{Discussion}

We have presented results from a community survey that assessed the acceptance of virtual conferencing technologies and other sustainability measures in the ALIFE community as well as the general scientific community. In summary, our results show that scientists are rather welcoming of almost all suggested measures that help us to lower the eco- logical impact of academic conferences. Measures are more accepted when they are presented as incentives rather than compulsion.

The most positive feedback was on the inclusion of remote participation by means of virtual talks and poster presentations, as well as making these presentations accessible via streaming and/or broadcasting. This is very encouraging, as it is arguably the most effective measure to reduce the ecological cost associated with conferencing, i.e., the impact of air travel.

At the same time, there is strong concern that virtual conferencing techniques can only deliver the scientific content that is presented, but fail to cover other important aspects of conferencing, primarily the opportunity for personal networking and the opportunity to fully engage in the conference. There is the associated fear that the spreading of virtual conferencing will deprive early career researchers from their professional opportunites. Quoting one of the survey respondents: "The young need to travel: for their own sake and for the sake of the elder." While we fully agree with this sentiment, we point out that opportunities for travel are already constrained by boundary conditions such as travel budget limitations, competing professional or personal commitments such as child care obligations, or disabilities. Virtualization has the potential to make scientific conferences more inclusive by lowering these barriers. In a time, where a growing number of academics experiences a personal conflict between their ecological ethos and a perceived duty to travel, virtual conferencing technologies can empower academics to decide more freely how often and how far to travel, without sacrificing their engagement with the community entirely.

The enforcement of presential participation quota has received the lowest acceptance among respondents, both within the ALIFE community as well as the wider scientific audience. This is unfortunate as it is the only proposed measure by which conference organizers can guarantee that their event does not exceed some given ecological impact target. Yet, the survey results reveal that academics are more receptive for such enforcements, if alternative remote participation comes with significant monetary incentives or is offered for free.

The results of this survey will be taken forward, and will be closely considered in the organization of ALIFE 2019. As this years' ALIFE conference will explore the theme "how Artificial Life can help solve societal challenges", we are eager to trial a variety of solutions toward low-carbon conferencing in the hope to make a noticable real-world difference. We understand that changing the habits of a community is an undertaking that can only show success, if done in line with the communities sentiments and agenda. Therefore, we regard our efforts merely as starting point of a long-term process that needs to be performed in discussion with the ALIFE community, the International Society of Artificial Life 
(ISAL), as well as future conference organizers. The presented survey is the start of this discussion.

\section{Acknowledgements}

We wish to express our gratitude to all participants of our online survey for the time taken, the depth of their considerations, and the many ideas they have provided.

\section{References}

Achten, W. M. J., Almeida, J., and Muys, B. (2013). Carbon footprint of science: More than flying. Ecological Indicators, 34:352-355.

Cobb, K. M., Kalmus, P., and Romps, D. M. (2018). AGU should support its members who fly less.

Girling, J., Liese, P., Dance, S., Gerbrandy, G.-J., Kyllönen, M., Eickhout, B., and dOrnano, M. (2018). Co2 emissions from aviation. Technical report, European Parliament.

Glover, A., Strengers, Y., and Lewis, T. (2017). The unsustainability of academic aeromobility in australian universities. Sustainability: Science, Practice and Policy, 13(1):1-12.

Hasan, M. (2018). New university rules encourage scientists to avoid air travel. https://www.wired.com/story/ climate-scientists-take-the-train/. Accessed: 2019-03-09.

IPCC (2018). Summary for policymakers. In Global Warming of $1.5^{\circ} \mathrm{C}$. An IPCC Special Report on the impacts of global warming of $1.5^{\circ} \mathrm{C}$ above pre-industrial levels and related global greenhouse gas emission pathways, in the context of strengthening the global response to the threat of climate change, sustainable development, and efforts to eradicate poverty. World Meteorological Organization, Geneva, Switzerland.

Krumdieck, S. and Orchard, S. (2011). Signs of change national networked e-conference: Highlighting emerging sustainability and social business. Social Business, 1(1):37-58.

Lee, D. S., Fahey, D. W., Forster, P. M., Newton, P. J., Wit, R. C., Lim, L. L., Owen, B., and Sausen, R. (2009). Aviation and global climate change in the 21 st century. Atmospheric Environment, 43(22-23):3520-3537.

Leybold-Johnson, I. (2019). Should academics be taking the plane for short trips? https://www. swissinfo.ch/eng/university-policies_ should-academics-be-taking-the-plane-for-short-trips-/ 44726904 . Accessed: 2019-03-09.

Nathans, J. and Sterling, P. (2016). Point of view: How scientists can reduce their carbon footprint. ELife, 5:e15928.

Nature editorial (2015). A clean, green science machine. Nature News, 519(7543):261.

Nevins, J. (2014). Academic jet-setting in a time of climate destabilization: Ecological privilege and professional geographic travel. The Professional Geographer, 66(2):298-310.

Wynes, S. and Donner, S. D. (2018). Addressing greenhouse gas emissions from business-related air travel at public institutions: a case study of the University of British Columbia. Technical report, Pacific Insitute for Climate Solutions. 